\title{
Heat and Mass Transfer of Vacuum Cooling for Porous Foods-Parameter Sensitivity Analysis
}

\author{
Zhijun Zhang, ${ }^{1}$ Yuekai Zhang, ${ }^{1}$ Tianyi Su, ${ }^{1}$ Wenhui Zhang, ${ }^{1}$ Lili Zhao, ${ }^{2}$ and Xun Li \\ ${ }^{1}$ School of Mechanical Engineering and Automation, Northeastern University, Shenyang 110004, China \\ ${ }^{2}$ School of Mechanical Engineering, Shenyang University, Shenyang 110044, China \\ Correspondence should be addressed to Zhijun Zhang; zhjzhang@mail.neu.edu.cn
}

Received 25 May 2014; Accepted 4 July 2014; Published 17 July 2014

Academic Editor: Jun Liu

Copyright (c) 2014 Zhijun Zhang et al. This is an open access article distributed under the Creative Commons Attribution License, which permits unrestricted use, distribution, and reproduction in any medium, provided the original work is properly cited.

\begin{abstract}
Based on the theory of heat and mass transfer, a coupled model for the porous food vacuum cooling process is constructed. Sensitivity analyses of the process to food density, thermal conductivity, specific heat, latent heat of evaporation, diameter of pores, mass transfer coefficient, viscosity of gas, and porosity were examined. The simulation results show that the food density would affect the vacuum cooling process but not the vacuum cooling end temperature. The surface temperature of food was slightly affected and the core temperature is not affected by the changed thermal conductivity. The core temperature and surface temperature are affected by the changed specific heat. The core temperature and surface temperature are affected by the changed latent heat of evaporation. The core temperature is affected by the diameter of pores. But the surface temperature is not affected obviously. The core temperature and surface temperature are not affected by the changed gas viscosity. The parameter sensitivity of mass transfer coefficient is obvious. The core temperature and surface temperature are affected by the changed mass transfer coefficient. In all the simulations, the end temperature of core and surface is not affected. The vacuum cooling process of porous medium is a process controlled by outside process.
\end{abstract}

\section{Introduction}

Vacuum cooling, a rapid cooling process, has huge ability to cool the porous food [1-5]. Its heat and mass transfer is a complicated process, which has been investigated by many researchers [6-17]. Jin et al. [6-8] developed and validated moisture movement model for vacuum cooling of cooked meat. The vacuum cooling of cooked meat with cylindrical shape was carried out to obtain the variations of temperature, moisture content, and evaporation rate. Sun et al. [9-13] developed a series of models of simultaneous transient heat and mass transfer with inner heat and mass generation for analyzing the performance of vacuum cooling cooked meats. In addition, a mathematical model is developed to analyze the performance of a vacuum cooler [14]. Dostal and Petera [15] gave a simple mathematical model of the vacuum cooling process which enables the prediction of a temperature evolution regarding an equipment size, vacuum pump parameters, and properties of the cooled liquid. He and Li [16] developed a model for predicting the temporal temperature and mass of spherical solid foods during vacuum cooling. They discuss the effects of product thermophysical properties, convection heat transfer coefficient, latent heat of evaporation, and vacuum environmental parameters that govern the heat and mass transfer of product. The temporal trends of total system pressure, product temperature such as surface temperature, center temperature, and mass-average temperature, and the mass of product were predicted. Compared to the theory study, the vacuum cooling has been used for many kinds of food, like ham [17], chicken breast [18, 19], beef [20-23], potato [24], cherry [25], pork [26, 27], mussels [28], carrot [29], rose [30,31], purslane [32], lettuce [33], and others [1-4].

Compared with the experiment, the simulation shows the excellent ability in time and economic. Except the model theory and method, the parameter of model is also the basic element which decided the results accuracy with the real physical process. The parameter is very difficlt to gotten by 


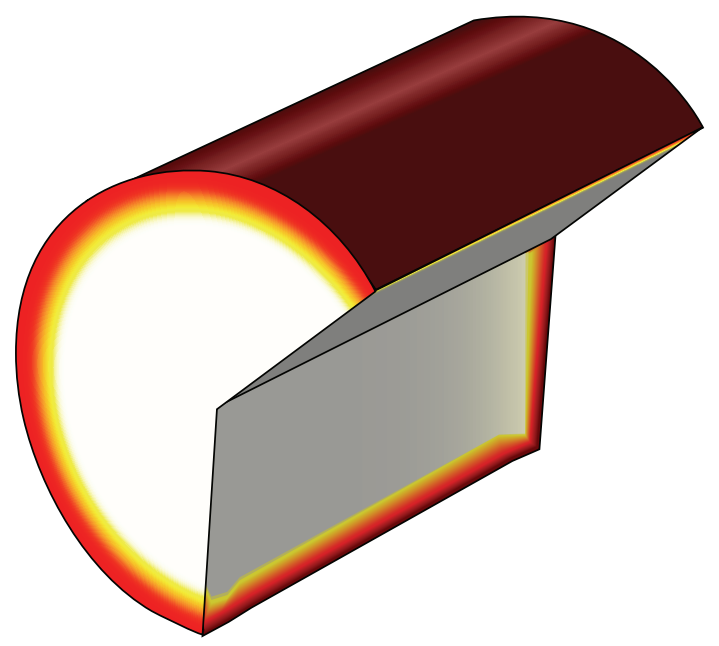

FIGURE 1: 2D axis symmetry model of porous food.

experiment. And most of them in modeling study were gotten from the other references that maybe not right in their present model. Few studies were focused on parameter sensitivity of the vacuum cooling process. In this paper, heat and mass transfer of porous food in the vacuum cooling process is implemented by using a nonequilibrium method. Sensitivity analyses of the process to food density, thermal conductivity, specific heat, latent heat of evaporation, diameter of pores, mass transfer coefficient, viscosity of gas, and porosity were examined.

\section{Model and Method}

A physical two-dimensional axis symmetry model that explains the vacuum cooling process is shown in Figure 1. The total length of the porous food is $310 \mathrm{~mm}$. The diameter is $130 \mathrm{~mm}$. In order to simplify the calculation, half of the model was used.

The porous food consists of a continuous rigid solid phase and a continuous gas phase which are considered as ideal gases because the process temperature and pressure are normal scope. For a mathematical description of the transport phenomenon in a porous medium, the paper adopts a continuum approach, wherein macroscopic partial differential equations are achieved through the volume averaging of the microscopic conservation laws. The value of any physical quantity at a point in space is given by its average value on the averaging volume centered at this point.

The moisture movement of the inner porous medium is vapor movement. The liquid water could be become vapor, and the vapor is moved by the pressure gradient. The heat and mass transfer theory could be found everywhere [6-17].

The compressibility effects of the solid phase are negligible, and the phase is homogeneous:

$$
\bar{\rho}_{m}=\text { cste }
$$

The gaseous phase is considered an ideal gas. This phase ensures that

$$
\bar{\rho}_{v}=\frac{m_{v} \bar{P}}{R \bar{T}} .
$$

Mass conservation equations are written for each component in each phase. Given that the solid phase is rigid, the following is given.

For vapor,

$$
\varphi \frac{\partial\left(\bar{\rho}_{v}\right)}{\partial t}+\nabla \cdot\left(\bar{\rho}_{v} \bar{V}_{v}\right)=\dot{I}
$$

where the gas velocity is given by

$$
\begin{gathered}
\bar{V}_{v}=-\frac{k_{v}}{\mu_{v}} \cdot \nabla \bar{P}, \\
k_{v}=\frac{d^{2}}{32} .
\end{gathered}
$$

By considering the hypothesis of the local thermal equilibrium, the energy conservation is reduced to a unique equation:

$$
\rho_{m} c \frac{\partial \bar{T}}{\partial t}-\nabla(k \cdot \nabla \bar{T})=-\lambda \cdot \dot{I} .
$$

B.C. for (3) on the symmetric surface is

$$
\frac{\partial \bar{P}}{\partial n}=0
$$

B.C. for (3) on the outer surface is

$$
P=\bar{P}_{\mathrm{vc}} \text {. }
$$

B.C. for (5) on the symmetric surface is

$$
\frac{\partial \bar{T}}{\partial n}=0 .
$$

B.C. for (5) on the outer surface is

$$
\begin{aligned}
& -k \frac{\partial T}{\partial n}=h_{r}\left(T_{s}-T_{\mathrm{vc}}\right)-\lambda \cdot \dot{m}_{s} \\
& h r=\sigma \varepsilon\left(T_{s}^{2}+T_{\mathrm{vc}}^{2}\right)\left(T_{s}+T_{\mathrm{vc}}\right) .
\end{aligned}
$$

The initial pressure of vapor is as follows.

I.C. for (3) is

$$
\bar{P}=P_{\text {sat }, 0} .
$$

The initial temperature of porous food is as follows.

I.C. for (5) is

$$
\bar{T}=T_{0} .
$$

The evaporation rate is a complex function of drying process in porous medium. The phase change can be formulated in two ways, equilibrium and nonequilibrium. Evaporation of water has been implemented using an equilibrium 
TABLE 1: Parameters used in the simulation process.

\begin{tabular}{lccc}
\hline Parameter & Value & Source & Unit \\
\hline$\rho_{v}$ & Ideal gas & & $\mathrm{Kg} \mathrm{m}^{-3}$ \\
$\rho_{m}$ & 1072 & {$[13]$} & $\mathrm{kg} \mathrm{m}^{-3}$ \\
$c$ & 3439 & {$[13]$} & $\mathrm{J} \mathrm{kg}^{-1} \mathrm{~K}^{-1}$ \\
$k$ & 0.59 & {$[13]$} & $\mathrm{W} \mathrm{m}^{-1} \mathrm{~K}^{-1}$ \\
$\mu_{v}$ & $9.6 \times 10^{-6}$ & {$[8]$} & $\mathrm{Pa} \mathrm{s}^{-2}$ \\
$h_{m}$ & $8.4 \times 10^{-7}$ & {$[13]$} & $\mathrm{kg} \mathrm{Pa}^{-2} \mathrm{~m}^{-2} \mathrm{~s}^{-1}$ \\
$\lambda$ & $2791.2 \times 10^{3}$ & {$[8]$} & $\mathrm{J} \mathrm{kg}^{-1}$ \\
$\varphi$ & 6 & {$[13]$} & $\%$ \\
$T_{0}$ & $74+273.15$ & {$[13]$} & $\mathrm{K}$ \\
$T_{\mathrm{vc}}$ & $25+273.15$ & {$[13]$} & $\mathrm{K}$ \\
$a_{W}$ & 1 & {$[13]$} & \\
RH & 0.75 & & $\mathrm{~m}$ \\
$d$ & $2.5 \times 10^{-3}$ & {$[13]$} & \\
\hline
\end{tabular}

formulation where water in the solid matrix is assumed to be in equilibrium with water vapor in the surrounding air. However, recent studies have shown that evaporation is not instantaneous and nonequilibrium exists during rapid evaporation between gas phase and liquid phase. Furthermore, the equations resulting from an equilibrium formulation cannot be implemented in any direct manner in the framework of most commercial software. The more general expressions of nonequilibrium evaporation rate used for modeling of phase change in porous media that is consistent with studies on pure water just mentioned are given by previous researches [6-17]:

$$
\begin{gathered}
\dot{I}=4 \frac{\varphi}{d} h_{m}\left(a_{W} P_{\text {sat }}-P\right), \\
\dot{m}_{s}=h_{m} a_{W} P_{\text {sat }}-\mathrm{RH} \cdot P_{\mathrm{vc}} .
\end{gathered}
$$

Here is a parameter signifying the rate constant of evaporation. The nonequilibrium formulation, given by (12), allows precisely this; that is, it can express the evaporation rate explicitly and therefore would be preferred in commercial software and is therefore used in our model.

The phase change rate of water could not be decided by any method for porous medium cooling. The rate constant parameter has the dimension of reciprocal time in which phase change occurs. A large value of signifies that phase change occurs in a small time. For the assumption of equilibrium, the rate of evaporating is infinitely large or phase change occurs instantaneously. A very high value of evaporating rate, however, makes the convergence of the numerical solution difficult.

The parameters value of the model is listed in Table 1. Sensitivity analyses of the process to food density, thermal conductivity, specific heat, latent heat of evaporation, diameter of pores, mass transfer coefficient, viscosity of gas, and porosity were then examined by changing the value $\pm 20 \%$.

\section{Numerical Simulation}

COMSOL Multiphysics 3.5a was used to solve the set of equations. COMSOL is advanced software used for modeling

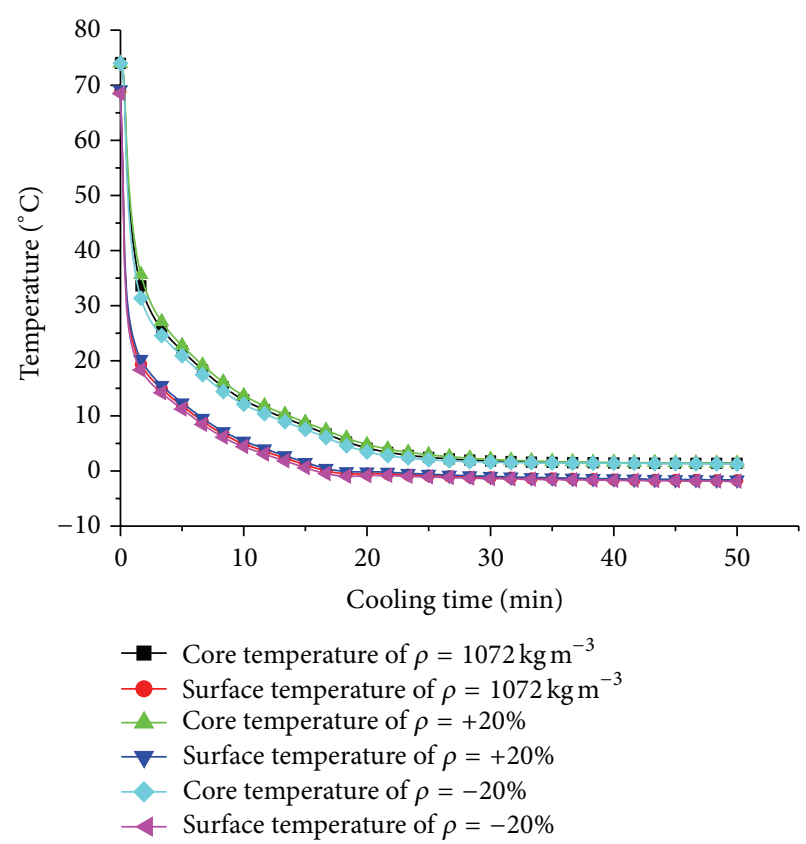

FIgURE 2: Sensitivity of vacuum cooling process to food density as measured by core and surface temperature.

and simulating any physical process described by partial derivative equations. The set of equations introduced above was solved using the relative initial and boundary conditions. COMSOL offers three possibilities for writing the equations: (1) a template (Fick Law, Fourier Law), (2) the coefficient form (for mildly nonlinear problems), and (3) the general form (for most nonlinear problems). Differential equations in the coefficient form were written using an unsymmetricalpattern multifrontal method. The paper used a direct solver for sparse matrices (UMFPACK), which involves significantly more complicated algorithms than solvers used for dense matrices. The main complication is the need to handle the fillin in factors $\mathrm{L}$ and $\mathrm{U}$ efficiently.

A two-dimensional (2D) axis symmetry grid was used to solve the equations using COMSOL Multiphysics 3.5a. The mesh consists of $25 \times 50$ elements (2D), and time stepping is free taken by solver. Several grid sensitivity tests were conducted to determine the sufficiency of the mesh scheme and to ensure that the results are grid independent. A backward differentiation formula was used to solve timedependent variables. Relative tolerance was set to $1 \times 10^{-4}$, whereas absolute tolerance was set to $1 \times 10^{-6}$. The simulations were performed using a Tongfang PC. It is using the Intel Core 2 Duo processor with $3.0 \mathrm{GHz}$ processing speed and $4096 \mathrm{MB}$ of RAM running by Windows 7 .

\section{Results and Discussion}

Figure 2 is the sensitivity of vacuum cooling process to food density as measured by core and surface temperature. The food density is changed with the cooling process because of the water evaporating. In [13], the density of food before cooling is $1072 \mathrm{~kg} \mathrm{~m}^{-3}$ but after cooling is $1024 \mathrm{~kg} \mathrm{~m}^{-3}$. But 


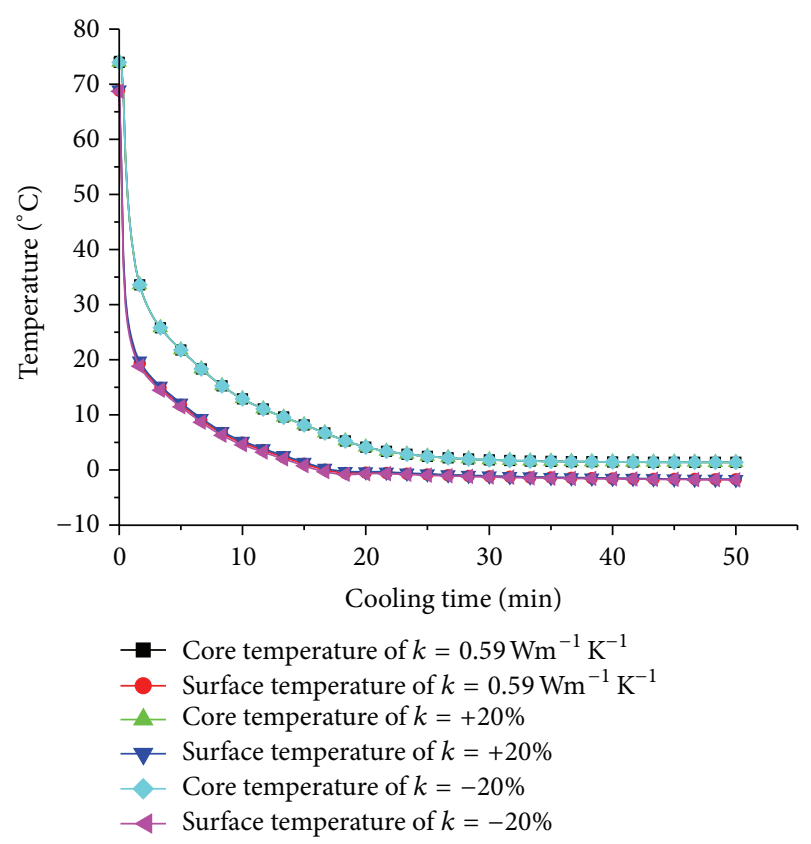

Figure 3: Sensitivity of vacuum cooling process to food thermal conductivity as measured by core and surface temperature.

its modeling does not discuss the effect of density change. In the present simulation, the density of food is increased $20 \%$ at first simulation. It is $1286 \mathrm{~kg} \mathrm{~m}^{-3}$. And then the density of food is lowered $20 \%$; it is $857.6 \mathrm{~kg} \mathrm{~m}^{-3}$. The simulation results show that the density increased, and the temperature is a little increased before the end pressure is gotten. The core temperature and surface temperature have the same change. The density would affect the vacuum cooling, but not the vacuum cooling end temperature. And in our simulation, the density is changed in $20 \%$, and it is a big scope in fact. So, we could conclude that the density sensitivity is not so big.

Figure 3 is the sensitivity of vacuum cooling process to food thermal conductivity as measured by core and surface temperature. The thermal conductivity is changed with the cooling process because of the water evaporating. In [13], the thermal conductivity of food before cooling is $0.59 \mathrm{~W} \mathrm{~m}^{-1} \mathrm{k}^{-1}$; after cooling it is $0.48 \mathrm{~W} \mathrm{~m}^{-1} \mathrm{k}^{-1}$. In [8], the thermal conductivity of food is gotten from reference by changed moisture and used the averaged thermal conductivity $0.4943 \mathrm{~W} \mathrm{~m}^{-1} \mathrm{k}^{-1}$. But its modeling is not discussed the effect of thermal conductivity change. In our simulation, the thermal conductivity of food is increased $20 \%$ at first. It is $0.708 \mathrm{~W} \mathrm{~m}^{-1} \mathrm{~K}^{-1}$. And then the thermal conductivity of food is lowered $20 \%$; it is $0.472 \mathrm{~W} \mathrm{~m}^{-1} \mathrm{~K}^{-1}$. The results show that the parameter sensitivity of thermal conductivity is very little. The surface temperature of food was slightly affected. The core temperature is not affected by the changed thermal conductivity. It is the process which is controlled by outside porous food that is vacuum condition.

Figure 4 is the sensitivity of vacuum cooling process to food specific heat as measured by core and surface temperature. The food specific heat is changed with the cooling process because of the water evaporating. In [13], the

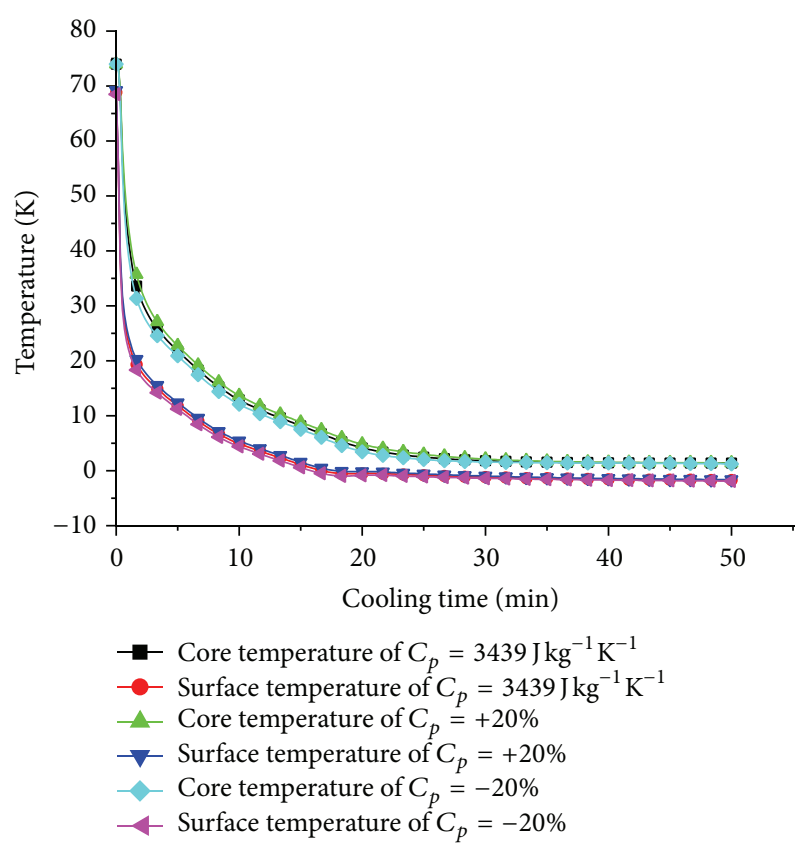

FIGURE 4: Sensitivity of vacuum cooling process to food specific heat as measured by core and surface temperature.

specific heat before cooling is $3439 \mathrm{~J} \mathrm{~kg}^{-1} \mathrm{~K}^{-1}$ after cooling is $3420 \mathrm{~J} \mathrm{~kg}^{-1} \mathrm{~K}^{-1}$. In [8], the specific heat is gotten from reference by changed moisture and used the averaged specific heat, $3214.8 \mathrm{~J} \mathrm{~kg}^{-1} \mathrm{~K}^{-1}$. But its modeling is not discussed the effect of specific heat change. In our simulation, the specific heat is increased $20 \%$ at first. It is $4126.8 \mathrm{~J} \mathrm{~kg}^{-1} \mathrm{~K}^{-1}$. And then the specific heat is lowered $20 \%$; it is $2751.2 \mathrm{~J} \mathrm{~kg}^{-1} \mathrm{~K}^{-1}$. The results show that the parameter sensitivity of specific heat is little, but obvious. The core temperature and surface temperature is affected by the changed specific heat. But the end temperature is not affected.

Figure 5 is the sensitivity of latent heat of evaporation in vacuum cooling process as measured by core and surface temperature. The latent heat of evaporation is changed with different food and material. In [11], the latent heat is given by the equation related temperature, but in [8], it is shown in Table 1. In the present simulation, the latent heat is increased $20 \%$ at first. It is $3349.44 . \mathrm{k} \mathrm{kg} \mathrm{k}^{-1}$. And then the latent heat is lowered $20 \%$; it is $2232.96 \mathrm{k} \mathrm{J} \mathrm{kg}^{-1}$. The results show that the parameter sensitivity of latent heat is little, but obvious. The core temperature and surface temperature are affected by the changed latent heat of evaporation. With the latent increase, the temperature is lowered. But the end temperature is not affected.

Figure 6 is the sensitivity of pores diameter in vacuum cooling process as measured by core and surface temperature. The pores diameter is material property. Different food should have the different diameter. But in $[8,13]$, the value is the same. It is $2.5 \mathrm{~mm}$. But the effect of pores diameter is not discussed in its modeling. In the present simulation, the pores diameter is increased $20 \%$ at first. It is $3 \mathrm{~mm}$. And then the pores diameter is lowered $20 \%$; it is $2 \mathrm{~mm}$. The results show that the parameter sensitivity of pores diameter is little, but 


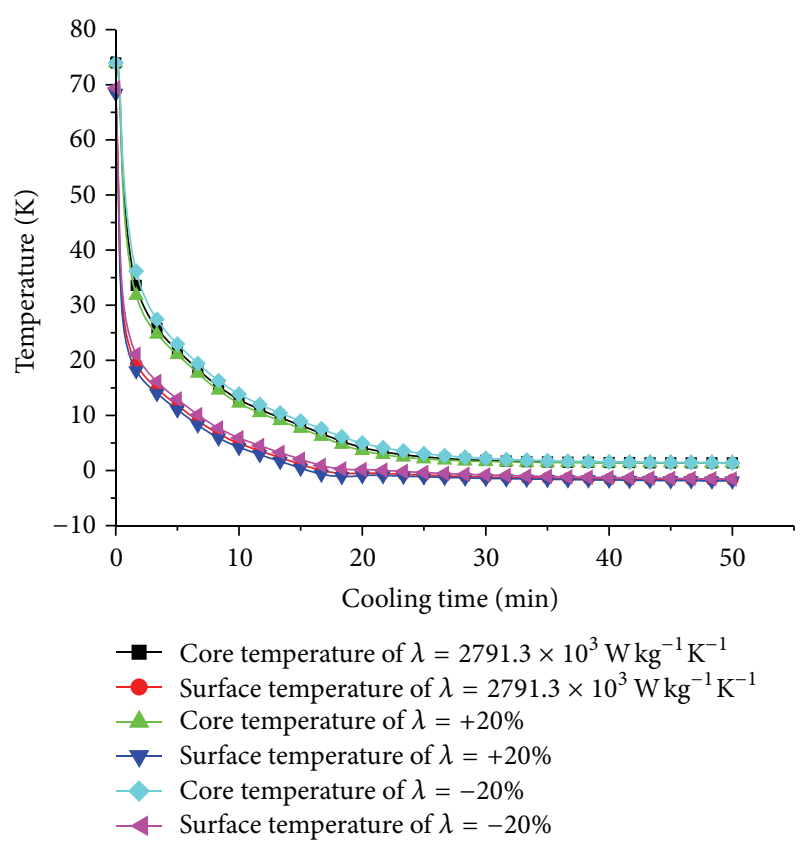

FIGURE 5: Sensitivity of vacuum cooling process to latent heat of evaporation as measured by core and surface temperature.

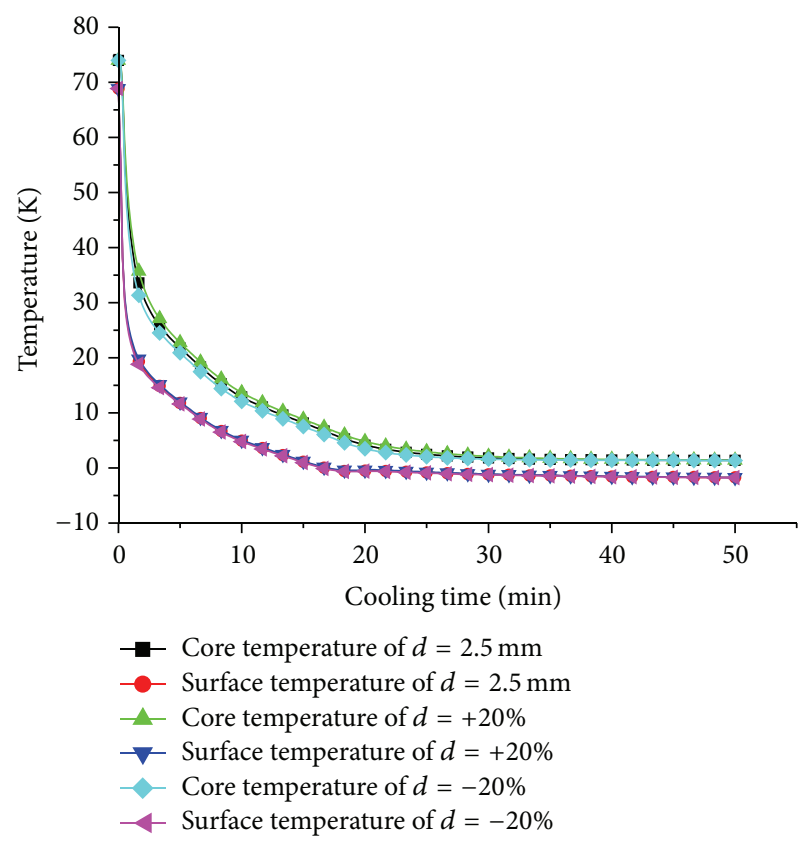

FIGURE 6: Sensitivity of vacuum cooling process to diameter of pores as measured by core and surface temperature.

obvious. The core temperature is affected by the diameter of pores. But the surface temperature is not affected obviously.

Figure 7 is the sensitivity of food porosity in vacuum cooling process as measured by core and surface temperature. The porosity is material property. Different food should have the different porosity. But in $[8,13]$, the value is the same. But the effect of porosity is not discussed in its modeling. In the present simulation, the porosity is increased $20 \%$ at first. It is

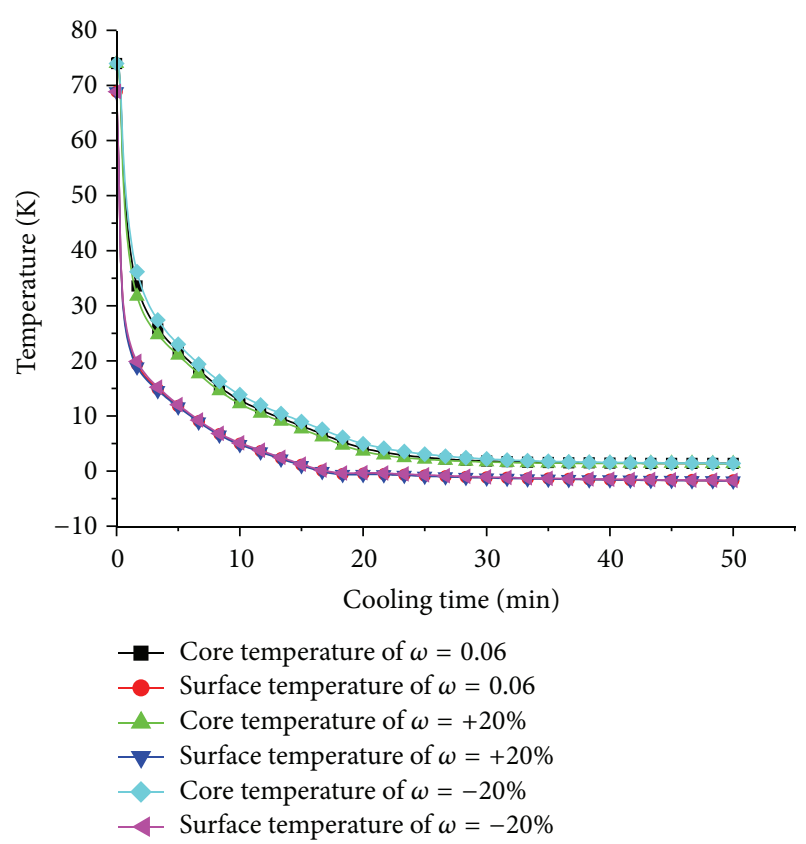

FIGURE 7: Sensitivity of vacuum cooling process to porosity as measured by core and surface temperature.

$7.2 \%$. And then the porosity is lowered $20 \%$; it is $4.8 \%$. The results show that the parameter sensitivity of porosity is little, but obvious. The core temperature is affected by the diameter of pores. But the surface temperature is not affected obviously.

Figure 8 is the sensitivity of gas viscosity in vacuum cooling process as measured by core and surface temperature. The gas viscosity should be measured in experiment, but most of them are gotten from reference. But the effect of gas viscosity is not discussed in its modeling. In our simulation, the gas viscosity is increased $20 \%$ at first. It is $11.52 \times 10^{-6} \mathrm{~Pa} \mathrm{~s}$. And then the specific heat is lowered $20 \%$; it is $7.68 \times 10^{-6} \mathrm{~Pa} \mathrm{~s}$. The results show that the parameter sensitivity of gas viscosity is so little, but not obvious. The core temperature and surface temperature is not affected by the changed gas viscosity. The end temperature is not affected.

Figure 9 is the sensitivity of mass transfer coefficient in vacuum cooling process as measured by core and surface temperature. The mass transfer coefficient is changed with the cooling process because the water evaporating is changed with the vacuum condition. But the effect of mass transfer coefficient is not discussed in its modeling. In our simulation, the mass transfer coefficient is increased $20 \%$ at first. It is $10.08 \times 10^{-7} \mathrm{~kg} \mathrm{~Pa}^{-2} \mathrm{~m}^{-2} \mathrm{~s}^{-1}$. And then the mass transfer coefficient is lowered $20 \%$; it is $6.728 .4 \times 10^{-7} \mathrm{~kg} \mathrm{~Pa}^{-2} \mathrm{~m}^{-2} \mathrm{~s}^{-1}$. The results show that the parameter sensitivity of mass transfer coefficient is obvious. The core temperature and surface temperature is affected by the changed mass transfer coefficient changed.

\section{Conclusion}

A coupled model of porous food vacuum cooling based on the theory of heat and mass transfer was implemented in 


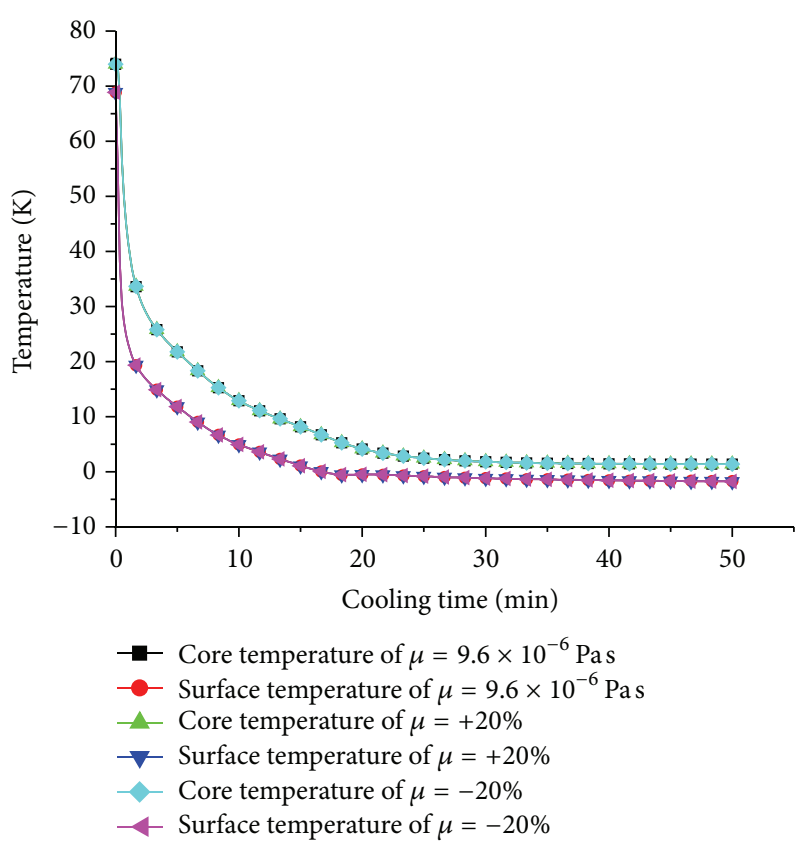

FIGURE 8: Sensitivity of vacuum cooling process to gas viscosity of as measured by core and surface temperature.

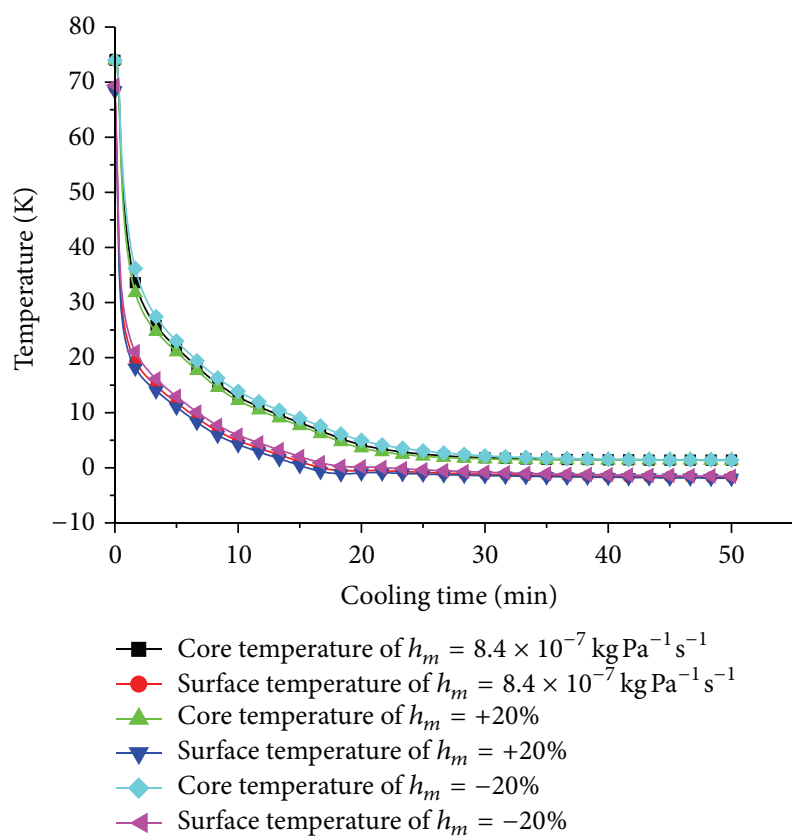

FIGURE 9: Sensitivity of vacuum cooling process to mass transfer coefficient as measured by core and surface temperature.

this paper. Sensitivity analyses of the process to food density, thermal conductivity, specific heat, latent heat of evaporation, diameter of pores, mass transfer coefficient, viscosity of gas, and porosity were then examined. The simulation results show that the density would affect the vacuum cooling process, but not the vacuum cooling end temperature. The parameter sensitivity of thermal conductivity is very little. The surface temperature of food was slightly affected. The core temperature is not affected by the changed thermal conductivity. The parameter sensitivity of specific heat is little, but obvious. The core temperature and surface temperature are affected by the specific heat changed. The parameter sensitivity of latent heat is little, but obvious. The core temperature and surface temperature are affected by the changed latent heat of evaporation. With the latent increase, the temperature is lowered. The parameter sensitivity of pores diameter is little, but obvious. The core temperature is affected by the diameter of pores. But the surface temperature is not affected obviously. The parameter sensitivity of porosity is little, but obvious. The core temperature is affected by the diameter of pores. But the surface temperature is not affected obviously. The parameter sensitivity of gas viscosity is so little, but not obvious. The core temperature and surface temperature is not affected by the changed gas viscosity. The parameter sensitivity of mass transfer coefficient is obvious. The core temperature and surface temperature is affected by the changed mass transfer coefficient. In all the simulation, the end temperature of core and surface is not affected. Compared with all the results, the sensitivity of parameters is not so big. It could conclude further that the vacuum cooling process is the process which is controlled by vacuum chamber condition.

\section{Nomenclature}

$a_{W}$ : Water activity

$c: \quad$ Specific heat $\left(\mathrm{J} \mathrm{kg}^{-1} \mathrm{~K}^{-1}\right)$

$d$ : Diameter of pores $(\mathrm{m})$

$h_{m}$ : Evaporation rate $\left(\mathrm{kg} \mathrm{Pa}^{-1} \mathrm{~m}^{-1} \mathrm{~s}^{-1}\right)$

$h_{r}$ : Heat transfer coefficient $\left(\mathrm{W} \mathrm{m}^{-2} \mathrm{~K}^{-1}\right)$

$\dot{I}: \quad$ Water phase rate $\left(\mathrm{kg} \mathrm{s}^{-1} \mathrm{~m}^{-3}\right)$

$k$ : Thermal conductivity $\left(\mathrm{W} \mathrm{m}^{-1} \mathrm{~K}^{-1}\right)$

$k_{v}: \quad$ Permeability $\left(\mathrm{m}^{2}\right)$

$m_{v}$ : Vapor molecular mass $\left(\mathrm{g} \mathrm{mol}^{-1}\right)$

$m_{s}: \quad$ Mass transfer coefficient $\left(\mathrm{kg} \mathrm{m}^{-2} \mathrm{~Pa}^{-1} \mathrm{~s}^{-1}\right)$

$n: \quad$ Outer unit normal to the product

$\bar{P}: \quad$ Pressure $(\mathrm{Pa})$

$P_{\text {sat }}:$ Vapor saturation pressure $(\mathrm{Pa})$

$P_{\text {sat }, 0}:$ Initial vapor saturation pressure $(\mathrm{Pa})$

$R: \quad$ Universal gas constant $\left(\mathrm{J} \mathrm{kmol}^{-1} \mathrm{~K}^{-1}\right)$

RH: Relative humidity

$t: \quad$ Time (s)

$\bar{T}: \quad$ Food temperature (K)

$T_{0}: \quad$ Initial temperature $(\mathrm{K})$

$T_{\mathrm{vc}}$ : Vacuum chamber temperature $(\mathrm{K})$

$T_{s}: \quad$ Surface temperature of food (K)

$\vec{V}_{v}:$ Vapor velocity $\left(\mathrm{m} \mathrm{s}^{-1}\right)$

$\varphi$ : $\quad$ Porosity (\%)

$\mu_{v}: \quad \operatorname{Viscosity}\left(\mathrm{kg} \mathrm{m}^{-1} \mathrm{~s}^{-1}\right)$

$\lambda$ : $\quad$ Latent heat of evaporation $\left(\mathrm{J} \mathrm{kg}^{-1}\right)$

$\bar{\rho}_{m}: \quad$ Food density $\left(\mathrm{kg} \mathrm{m}^{-3}\right)$

$\bar{\rho}_{v}$ : Vapor density $\left(\mathrm{kg} \mathrm{m}^{-3}\right)$. 


\section{Conflict of Interests}

The authors declare that there is no conflict of interests regarding the publication of this paper.

\section{Acknowledgments}

This research was supported by National Natural Science Foundation of China (Grant nos. 31000665, 51176027, 31371873, and 31300408) and the Fundamental Research Funds for the Central Universities of China (Grant no. N130403001).

\section{References}

[1] C. Feng, L. Drummond, Z. Zhang, D. Sun, and Q. Wang, "Vacuum cooling of meat products: current state-of-the-art research advances," Critical Reviews in Food Science and Nutrition, vol. 52, no. 11, pp. 1024-1038, 2012.

[2] D. Sun and L. Zheng, "Vacuum cooling technology for the agri-food industry: past, present and future," Journal of Food Engineering, vol. 77, no. 2, pp. 203-214, 2006.

[3] L. Zheng and D.-W. Sun, "Vacuum cooling for the food industry-a review of recent research advances," Trends in Food Science \& Technology, vol. 15, no. 12, pp. 555-568, 2004.

[4] K. McDonald and D. Sun, "Vacuum cooling technology for the food processing industry: a review," Journal of Food Engineering, vol. 45, no. 2, pp. 55-65, 2000.

[5] C. Cogné, P. U. Nguyen, J. L. Lanoisellé, E. van Hecke, and D. Clausse, "Modeling heat and mass transfer during vacuum freezing of puree droplet," International Journal of Refrigeration, vol. 36, no. 4, pp. 1319-1326, 2013.

[6] T. Jin, G. Li, Y. Gong, Y. Lu, and Y. Shi, "Modeling evaporationboiling phenomena during vacuum cooling of cooked meat," Intelligent Automation and Soft Computing, vol. 16, no. 6, pp. 1119-1133, 2010.

[7] T. X. Jin and L. Xu, "Development and validation of moisture movement model for vacuum cooling of cooked meat," Journal of Food Engineering, vol. 75, no. 3, pp. 333-339, 2006.

[8] T. X. Jin and L. Xu, "Numerical study on the performance of vacuum cooler and evaporation-boiling phenomena during vacuum cooling of cooked meat," Energy Conversion and Management, vol. 47, no. 13-14, pp. 1830-1842, 2006.

[9] D. W. Sun and L. J. Wang, "Development of a mathematical model for vacuum cooling of cooked meats," Journal of Food Engineering, vol. 77, no. 3, pp. 379-385, 2006.

[10] L. J. Wang and D. W. Sun, "Numerical analysis of the threedimensional mass and heat transfer with inner moisture evaporation in porous cooked meat joints during vacuum cooling," Transactions of the ASAE, vol. 46, no. 1, pp. 107-115, 2003.

[11] D. Sun and Z. Hu, "CFD simulation of coupled heat and mass transfer through porous foods during vacuum cooling process," International Journal of Refrigeration, vol. 26, no. 1, pp. 19-27, 2003.

[12] Z. H. Hu and D. W. Sun, "Corrigendum to "CFD predicting the effects of various parameters on core temperature and weight loss profiles of cooked meat during vacuum cooling": [Comput. Electron. Agric. 34 (2002) 111-127]," Computers and Electronics in Agriculture, vol. 39, no. 3, p. 255, 2003.

[13] L. Wang and D. Sun, "Modelling vacuum cooling process of cooked meat-part 2: mass and heat transfer of cooked meat under vacuum pressure," International Journal of Refrigeration, vol. 25, no. 7, pp. 862-871, 2002.

[14] L. J. Wang and D. W. Sun, "Modelling vacuum cooling process of cooked meat-part 1: analysis of vacuum cooling system," International Journal of Refrigeration, vol. 25, no. 7, pp. 854-861, 2002.

[15] M. Dostal and K. Petera, "Vacuum cooling of liquids: mathematical model," Journal of Food Engineering, vol. 61, no. 4, pp. 533-539, 2004

[16] S. Y. He and Y. F. Li, "Theoretical simulation of vacuum cooling of spherical foods," Applied Thermal Engineering, vol. 23, no. 12, pp. 1489-1501, 2003.

[17] X. Y. Song and B. L. Liu, "The optimization of volumetric displacement can uniformize the temperature distribution of heated ham during a vacuum cooling process," Food Science and Technology Research, vol. 20, no. 1, pp. 43-49, 2014.

[18] F. C. Schmidt and J. B. Laurindo, "Alternative processing strategies to reduce the weight loss of cooked chicken breast fillets subjected to vacuum cooling," Journal of Food Engineering, vol. 128, pp. 10-16, 2014.

[19] F. C. Schmidt, G. M. F. Aragão, and J. B. Laurindo, "Integrated cooking and vacuum cooling of chicken breast cuts in a single vessel," Journal of Food Engineering, vol. 100, no. 2, pp. 219-224, 2010.

[20] Z. Zhang, L. Drummond, and D. Sun, "Vacuum cooling in bulk of beef pieces of different sizes and shape-evaluation and comparison to conventional cooling methods," Journal of Food Engineering, vol. 116, no. 2, pp. 581-587, 2013.

[21] L. Drummond and D. W. Sun, "Evaluation of the immersion vacuum cooling of cooked beef joints-mathematical simulation of variations in beef size and porosity and pressure reduction rates," Innovative Food Science \& Emerging Technologies, vol. 16, pp. 205-210, 2012.

[22] L. Drummond, D. Sun, C. T. Vila, and A. G. M. Scannell, "Application of immersion vacuum cooling to water-cooked beef joints: quality and safety assessment," LWT: Food Science and Technology, vol. 42, no. 1, pp. 332-337, 2009.

[23] L. Drummond and D. Sun, "Temperature evolution and mass losses during immersion vacuum cooling of cooked beef joints-a finite difference model," Meat Science, vol. 80, no. 3, pp. 885-891, 2008.

[24] L. G. G. Rodrigues, D. Cavalheiro, F. C. Schmidt, and J. B. Laurindo, "Possibilities for integrating cooking and vacuum cooling of potatoes in the same vessel," Journal of Food Processing and Preservation, vol. 37, pp. 846-854, 2013.

[25] S. Y. He, G. C. Zhang, Y. Q. Yu, R. G. Li, and Q. R. Yang, "Effects of vacuum cooling on the enzymatic antioxidant system of cherry and inhibition of surface-borne pathogens," International Journal of Refrigeration, vol. 36, no. 8, pp. 23872394, 2013

[26] C. Feng, L. Drummond, Z. Zhang, and D. Sun, "Effects of processing parameters on immersion vacuum cooling time and physico-chemical properties of pork hams," Meat Science, vol. 95, no. 2, pp. 425-432, 2013.

[27] X. Dong, H. Chen, Y. Liu, R. Dai, and X. Li, "Feasibility assessment of vacuum cooling followed by immersion vacuum cooling on water-cooked pork," Meat Science, vol. 90, no. 1, pp. 199-203, 2012.

[28] D. Cavalheiro, F. C. Schmidt, L. G. G. Rodrigues, C. Siga, F. Leitempergher, and J. B. Laurindo, "Processing of Perna Perna mussels using integrated process of cooking and vacuum 
cooling," Journal of Food Process Engineering, vol. 36, no. 2, pp. 192-201, 2013.

[29] L. G. G. Rodrigues, D. Cavalheiro, F. C. Schmidt, and J. B. Laurindo, "Integration of cooking and vacuum cooling of carrots in a same vessel," Ciencia e Tecnologia de Alimentos, vol. 32, no. 1, pp. 187-195, 2012.

[30] X. Y. Song, W. S. Wang, C. Zhang, Q. Ma, and Y. F. Li, "Postharvest physiochemical responses of cut rose (Rosa hybrida L.) to antitranspirant and vacuum cooling," Philippine Agricultural Scientist, vol. 94, no. 4, p. 368, 2011.

[31] H. M. Ozturk and H. K. Ozturk, "Effect of pressure on the vacuum cooling of iceberg lettuce," International Journal of Refrigeration, vol. 32, no. 3, pp. 395-403, 2009.

[32] H. Mutlu Ozturk, H. K. Ozturk, and G. Kocar, "Comparison of vacuum cooling with conventional cooling for purslane," International Journal of Food Engineering, vol. 7, no. 6, 2011.

[33] S. Y. He and Y. Li, "Experimental study and process parameters analysis on the vacuum cooling of iceberg lettuce," Energy Conversion and Management, vol. 49, no. 10, pp. 2720-2726, 2008. 


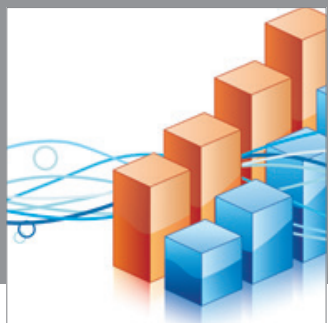

Advances in

Operations Research

mansans

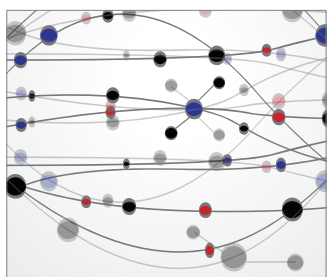

The Scientific World Journal
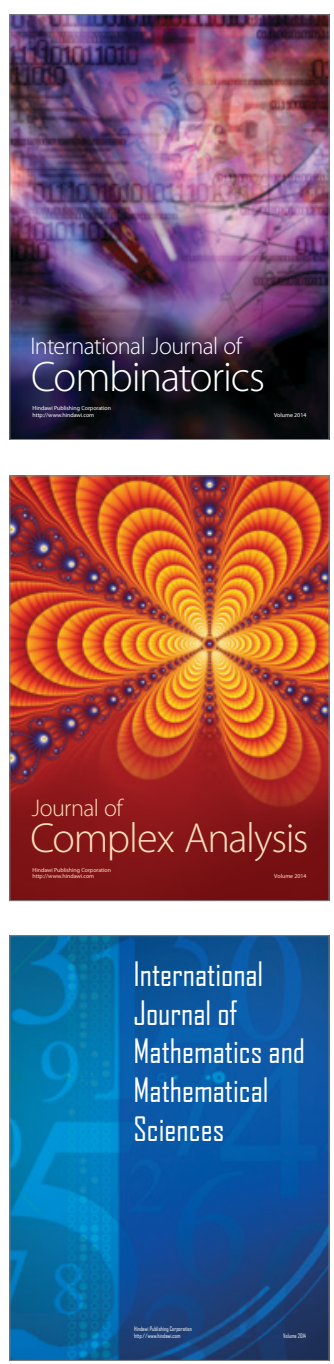
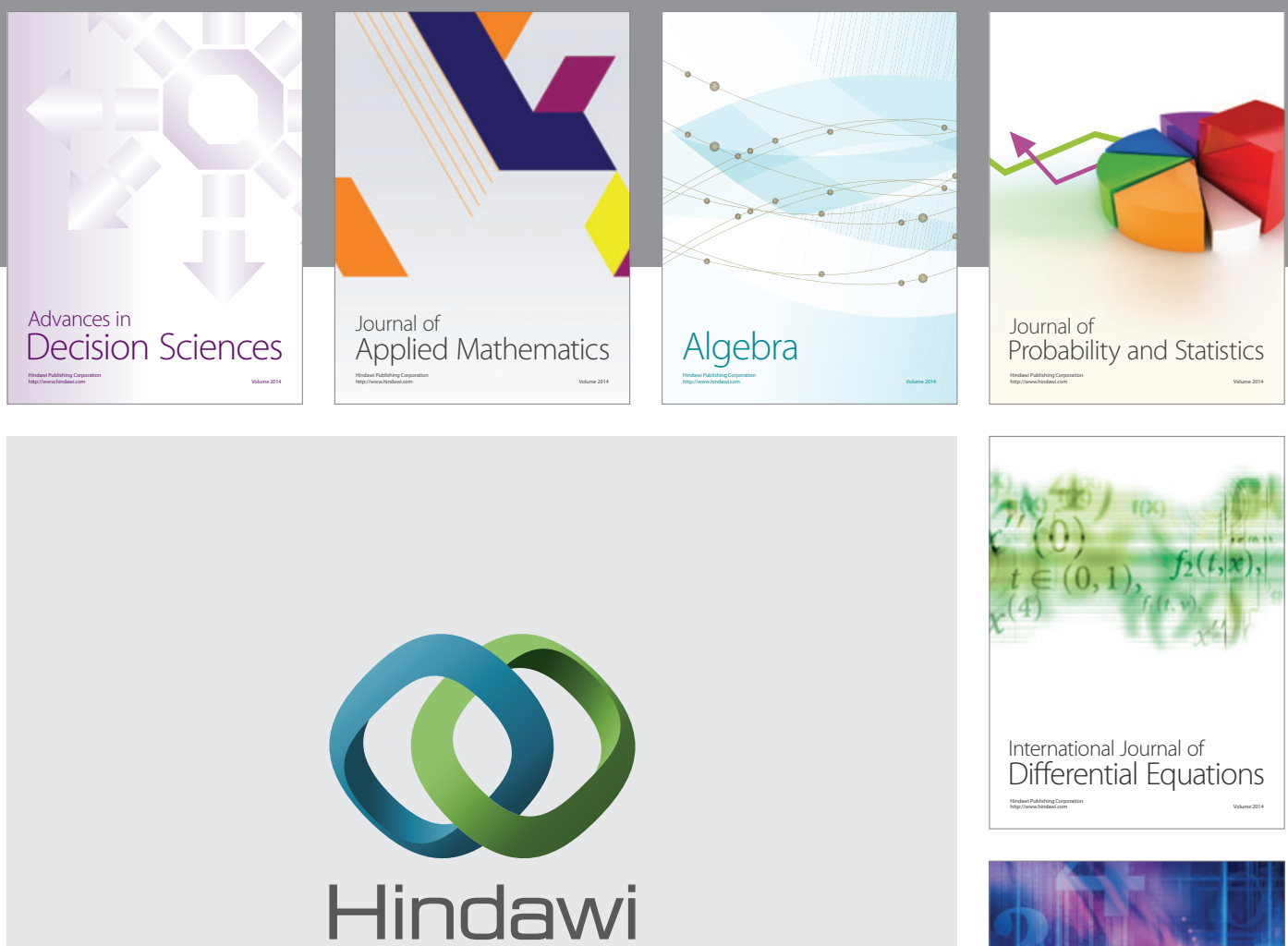

Submit your manuscripts at http://www.hindawi.com
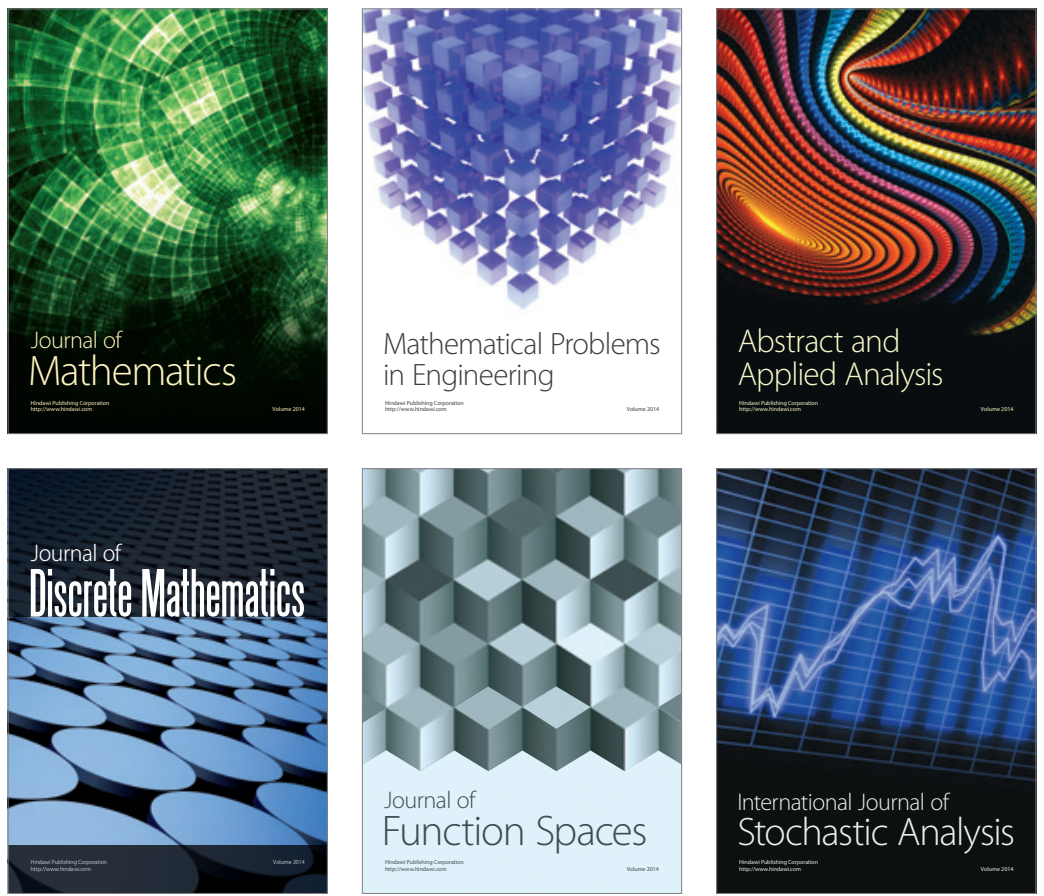

Journal of

Function Spaces

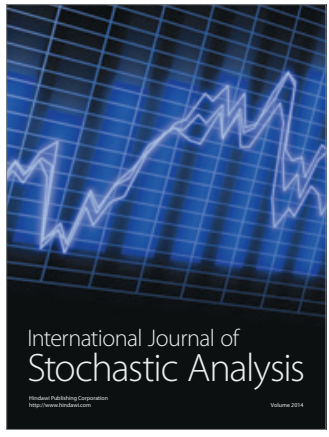

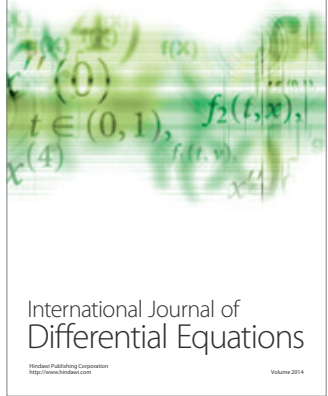
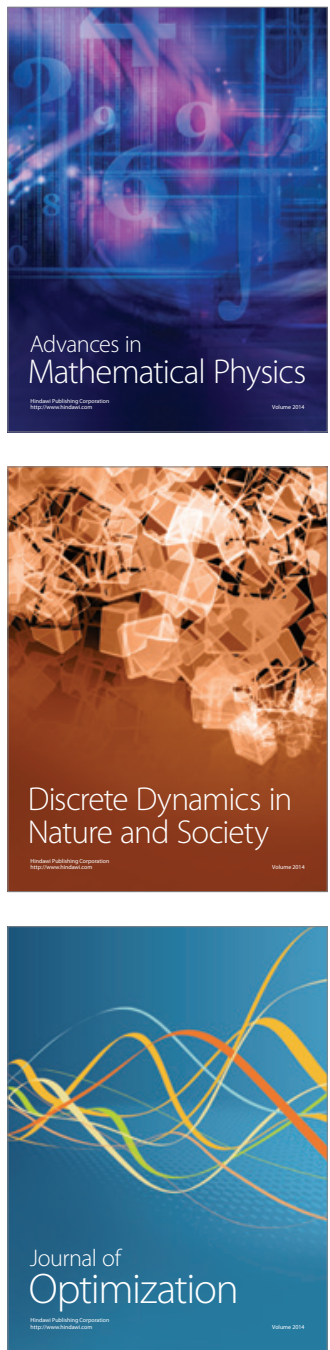